\title{
New correlated phenomena in magic-angle twisted bilayer graphene/s
}

\section{Tuning superconductivity in twisted bilayer graphene}

Authors: Matthew Yankowitz, Shaowen Chen, Hryhoriy Polshyn, K. Watanabe, T. Taniguchi, David Graf, Andrea F. Young, Cory R. Dean

Science 24, Jan 2019

\section{Emergent ferromagnetism near three-quarters filling in twisted bilayer graphene}

Authors: Aaron L. Sharpe, Eli J. Fox, Arthur W. Barnard, Joe Finney, Kenji

Watanabe, Takashi Taniguchi, M. A. Kastner, David Goldhaber-Gordon

arXiv:1901.03520, Jan 2019

\section{Cascade of superconducting domes and magnetic order around quarter} filling in magic angle bilayer graphene Authors: Dmitri Efetov, ICFO - Institut de Ciencies Fotoniques

KITP Correlations in Moire Flat Bands program, Jan 222019

\section{Recommended with a Commentary by Michael Zaletel, University of California, Berkeley}

Since the discovery of correlated insulators and superconductivity in magic-angle twisted bilayer graphene (tBLG) ([1, 2], JCCM April 2018), theorists have been excitedly pursuing the alluring mix of band topology, symmetry breaking, Mott insulators and superconductivity at play, as well as the potential relation (if any) to high- $T_{c}$ physics. Now a new stream of experimental work is arriving which further enriches the story. To briefly recap Episodes 1 and 2 (JCCM April and November 2018), when two graphene layers are stacked with a small rotational mismatch $\theta$, the resulting long-wavelength moire pattern leads to a superlattice potential which reconstructs the low energy band structure. When $\theta$ approaches the "magic-angle" $\theta_{M} \sim 1^{\circ}$, the band structure features eight nearly-flat bands which fill when the electron number per moire unit cell, $n / n_{0}$, lies between $-4<n / n_{0}<4$. The bands can be counted as $8=2 \times 2 \times 2$ : for each spin $(2 \times)$ and valley $(2 \times)$ characteristic of monolayer graphene, tBLG has has $2 \times$ flat bands which cross at mini-Dirac points. Crudely speaking, the alignment angle $\theta$ controls the bandwidth / interaction ratio, $\left|\theta-\theta_{M}\right| \sim t / U$. The origin and topological intricacies of this band structure were the subject of JCCM November 2018. In addition to the insulating states at $n / n_{0}= \pm 4$ expected from the single-particle band gap, near the magic-angle Cao et. al. observed "correlated insulator" states at $n / n_{0}= \pm 2$ 
not expected from band-theory[1] and superconducting $(\mathrm{SC})$ domes $\left(T_{c}=1.7 \mathrm{~K}\right)$ for densities near the $n / n_{0} \sim-2 \pm \delta$ insulator.[2] These features are not present in large-bandwidth devices far from the magic-angle.

The (at least) surface-level similarity to the physics of "doping a Mott insulator" has not gone unnoticed, as evidenced by the many dozens of theoretical papers to date. But from an experimental point of view many basic facts remain unclear. Are the integers $n / n_{0}= \pm 2$ special, or are further correlated states waiting to be found in cleaner samples and different parameter regimes? Which of the symmetries, such as spin-rotation and time-reversal, are broken / present in the superconducting and insulating states? What is the pairing channel of the superconductor? How strong are electron-phonon interactions? Does the phenomenology depend on the substrate, or can the tBLG be analyzed in isolation?

A trio of new experimental results from Yankowitz et al., Sharpe et al., and the Efetov Lab have begun to answer some of these questions. First a brief overview. $n / n_{0}= \pm 2$ is no longer so special: aggregating across devices and including data at moderate fields $B \sim 0-4 \mathrm{~T}$, insulating features emanate from every integer $-4 \leq n / n_{0} \leq 4$, albeit with widely ranging energy scales. Furthermore, in the device of Efetov superconducting pockets are observed between many (at least half, and potentially all) of these integers. Finally, there appear to be qualitative differences between devices rather than a unified tBLG phase diagram. Most notably, in the device of Sharpe et. al., the $n / n_{0}=3$ insulator has a dramatic zerofield anomalous Hall response, spontaneously breaking time-reversal symmetry, while the Yankowitz and Efetov devices do not. They suggest the difference may arise from alignment with the hexagonal boron-nitride (hBN) substrate. The hBN-tBLG alignment angle, which isn't usually well-controlled or characterized, can modulate the tBLG band structure, and consequently there may be a family of magic-angle tBLGs with different phase diagrams. This should be kept in mind when attempting to synthesize observations across devices.

The ideal platform for studying flat-band physics would allow us to control $t / U$ in addition to $n$ and $T$. Yankowitz et al. show this can be done in-situ using pressure. The more obvious approach is to change $\theta$, but this requires building different devices, which, with current capabilities, leads to variations in disorder and other imperfections likely to swamp the quantitative effect of $t / U$. To overcome this obstacle Yankowitz instead use pressure to change $\theta_{M}$, which depends on the magnitude of the interlayer tunneling $w$ and intralayer tunneling $t_{0}$ roughly as $\theta_{M} \propto w / t_{0}$. Pressure increases $w$, and hence can be used to control $t / U \propto\left|\theta-\theta_{M}\right|$ in-situ. They find that the activation gaps of the insulators, as well as the $T_{c}$ of the superconductors, have a non-monotonic "dome"-like dependence on pressure (albeit with

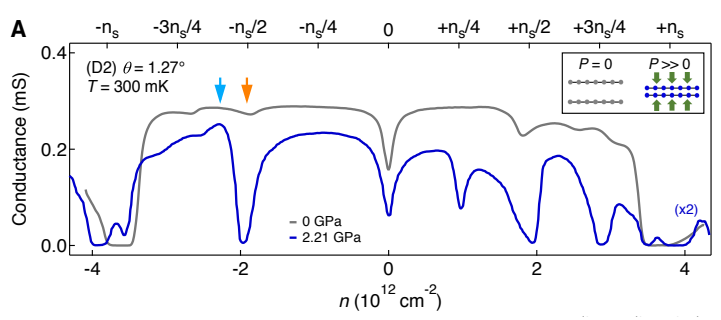

Figure 1: $\quad$ Conductance trace from Yankowitz, et al. across $-4 \leq n / n_{0} \leq 4$ at two difference pressures $P$. Holding fixed the tBLG alignment $\theta$, the magicangle $\theta_{M}$ is modulated by $P$, with correlated insulators appearing only near the putative magic-angle condition $\theta \sim \theta_{M}$ where the bandwidth is quenched. 3 data points) consistent with tuning across the theoretically-predicted flat-band condition. As an added benefit pressure increases $\theta_{M}$, thereby reducing the moire lattice constant and increasing the energy scale, with $\mathrm{SC} T_{c}$ up to $\sim 3 \mathrm{~K}$. 
They further observed a new correlated insulator at $n / n_{0}=3$, and where the original work of Cao et al. [2] found SC at $n / n_{0}=-2$, Yankowitz finds SC for $n / n_{0}= \pm 2$. By further mapping out the quantitative dependence of these insulators on the electric and magnetic field, the degeneracy of their Landau fans, and the Fraunhoffer patterns of the critical current, their work provides a rich set of constraints for theory.

The results of Sharpe et al. point to the potential role of topology in tBLG. In their device they observe resistance peaks at $n / n_{0}=2,3$, but no superconductivity. Remarkably, at $n / n_{0}=3$ the Hall resistance shows hysteresis in $R_{x y}$ about $B_{\perp}=0$, Fig. 2, indicating that time-reversal is spontaneously broken, with a zero-field value which is a sizable fraction of $h / e^{2}$. While not a quantized anomalous Hall (QAH) insulator, it may become one with further device improvements, making tBLG an unexpected platform for topological QAH physics. Non-local conductance measurements further support the presence of edge transport.

A spontaneous AH effect was not seen in the earlier experiments, so what makes their device different? Sharpe et al. provide an important clue. In addition to the resistance peaks tied to integer values of $n / n_{0}$, they observe a second resistance peak at $n / n_{0}=4.6$. They attribute this to a second moire-pattern between the tBLG and a nearly-aligned hBN substrate on one side of the device. This interpretation is borne out by optical imaging, as well as by a strong asymmetry in the response of the insulators to a perpendicular electric field under $E_{\perp} \leftrightarrow-E_{\perp}$, which is hard to explain without a substrate interaction since isolated tBLG has a symmetry which exchanges the layers. The Yankowitz device shows similar, though somewhat less pronounced asymmetry. As a control experiment, they test a second device which was carefully confirmed to be highly hBN-unaligned on both sides, and no

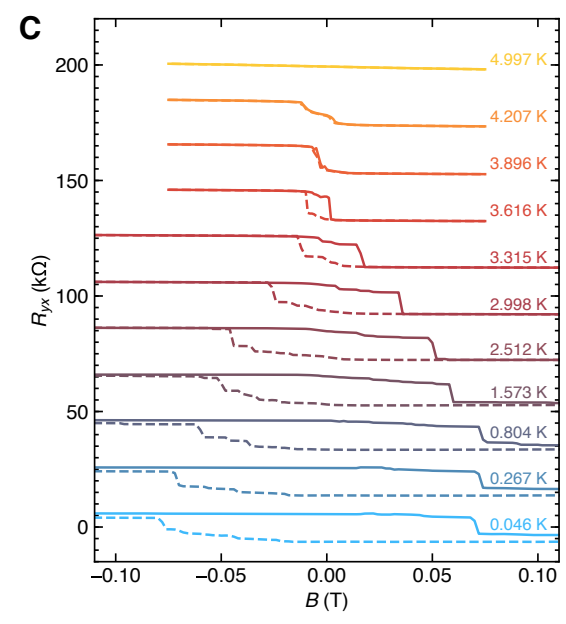

Figure 2: Hall resistance $R_{x y}$ at $n / n_{0} \sim 3$ in the tBLG device of Sharpe, et. al, as a function of out-of-plane field $B$. Below $T \sim 4 \mathrm{~K}$ a hysteresis loop develops, consistent with spontaneous breaking of time-reversal symmetry at $B=0$. The resistance $R_{x y} \sim 6-11 \mathrm{k} \Omega$ is of order the inverse conductance quantum but not obviously quantized.

AH physics is found. It seems clear then that the substrate has an important effect on the physics.

As Sharpe et al. point out, the relation between hBN-alignment and an anomalous Hall effect is not a complete mystery. The mini-Dirac points are protected by $C_{2} T$ (a 180-degree rotation combined with time-reversal). But the hBN coupling is known to break $C_{2} T$, with a magnitude which increases with the degree of hBN-tBLG alignment. If $C_{2} T$ is broken a band-gap will open at neutrality, and recent theoretical calculations show the resulting bands carry non-zero Chern number $C= \pm 1$. Spontaneous partial / full polarization into one of these bands would then lead to a AH / QAH effect. What is a mystery is how their finding relates to the earlier observations of superconductivity, which is not observed in this device. Are superconductivity and the (potential) Chern-bands antagonists - a possible clue regarding the nature of the pairing - or will further experiments show their union? 
The preliminary results of Efetov's Lab have so far been reported only at the KITP Moire Flat Bands program, but are striking enough to require mentioning here. An important theme across the conference was the ubiquity of twist-angle disorder (slow modulations in $\theta$ across the device), which blurs out $n / n_{0}$ because $\theta$ determines $n_{0}$. The Efetov device was reported to have very low twist-angle disorder $\left(\Delta \theta \sim 0.01^{\circ}\right)$, and featured a multitude of new insulators and superconductors (Fig. 3). They are the first to report an activated insulator $(\Delta \sim 1.5 \mathrm{meV})$ at $n / n_{0}=0$, perhaps because of the aforementioned effect of hBN, though the device is not thought to be strongly hBN-aligned. [3] At $B_{\perp}=0$ activated insulating gaps are reported at $n / n_{0}=-4,-2,0,1,2,3,4$, with
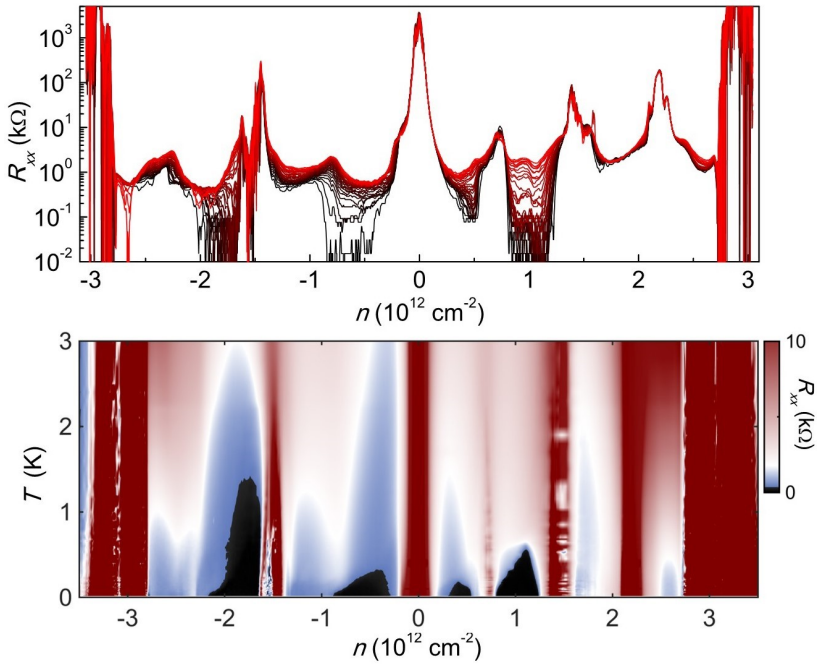

Figure 3: Resistivity traces for $-4 \leq n / n_{0} \leq$ 4 in the tBLG device of Efetov, et. al. Courtesy of Dmitri Efetov. low-resistance domes between every integer, four of which are consistent with $2 \mathrm{D}$ superconductivity with $T_{c} \sim 0.14-3 \mathrm{~K}$. The devices show further interesting behavior for $B_{\perp}>0$. At $n / n_{0}=3$, there is no $\mathrm{AH}$ behavior, in contrast to Sharpe. But for $n / n_{0}=-1$, at $B_{\perp} \sim 4 \mathrm{~T}$ there is a first-order jump in both $R_{x y}$ and $R_{x x}$ with clear hysteresis. While not the spontaneous-symmetry breaking found by Sharpe et al., it is certainly suggestive of a similar anomalous Hall state and the lurking role of topology.

Efetov's results raise a linguistic question: does tBLG feature one superconducting dome to rule them all, interrupted by insulators, or a sequence of doped Mott insulators? A more precise question is whether the SCs inherit the putative symmetry breaking of the neighboring insulators, and if so what symmetries are broken. If the stream of experiments keeps apace we may soon have an answer.

\section{References}

[1] Correlated insulator behaviour at half-filling in magic-angle graphene superlattices, Y. Cao et al. Nature 556, 7699, 80. (2018)

[2] Unconventional superconductivity in magic-angle graphene superlattices, Y. Cao et al. Nature 556, 7699, 43. (2018).

[3] On the nature of the correlated insulator states in twisted bilayer graphene, Ming Xie and Allan MacDonald, arXiv:1812.04213 (2018). 